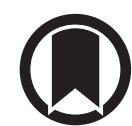

CrossMark

\title{
Impact of the SARS-CoV-2 epidemic on tuberculosis treatment outcome in Northern Italy
}

\author{
To the Editor:
}

We read with interest the letters by TADOLINI et al. [1] and STOchino et al. [2] in a recent issue of the European Respiratory Journal, which summarise the first available evidence on tuberculosis (TB) and coronavirus disease 2019 (COVID-19) [3].

The COVID-19 pandemic poses dramatic challenges to the capacity of the global healthcare system. As of 25 June, 2020, Brescia Province, in Lombardy, reported 15611 SARS-CoV-2 patients [4], accounting for $6.5 \%$ of all Italian cases, being one of the worst COVID-19 epicentres in Europe. The impact on healthcare delivery went far beyond the management of COVID-19 patients, as hospitals and health care facilities had to convert significant resources to its response.

Although overall reducing out-patient consultations, the Infectious and Tropical Diseases Department of the Spedali Civili of Brescia maintained all TB services unchanged and did not interrupt the cascade of TB care. We performed a retrospective, observational cohort analysis of the TB cascade of care among patients followed at the TB Clinic in Brescia in the period 1 March to 30 April, 2020 during the COVID-19 epidemic (study period) and compared them with those of March to April 2019 (control period). Data were censored at 30 June, 2020 and 2019, respectively. All patients who received a diagnosis of TB and who were receiving treatment for $\mathrm{TB}$, either followed in the out-patients $\mathrm{TB}$ clinic or admitted to the wards, were included in the analysis. Demographic, clinical and treatment outcome information were retrieved from paper and electronic (NetCare version 1.05.12; Abeni Alba Software) clinical charts. Outcome indicators were the number of new TB diagnoses, TB treatment outcome, and adherence to the scheduled medical visits. Categorical variables were described using frequencies and percentages, and Chi-squared test or exact Fisher test were applied. Statistical analysis was performed using one tailed test considering significant $\mathrm{p}$-values $<0.05$.

Overall, 65 patients entered TB care during the study period, compared to 76 in the control period. The diagnosis was based on microbiological criteria in $52(80 \%)$ and 69 (79\%) patients, respectively. There were no differences in age, gender, and proportion of foreign-born people during the two periods. In both periods there were three cases of multidrug-resistant $\mathrm{TB}$, one case of extensively drug resistant $\mathrm{TB}$ (XDR-TB) and one pre-XDR TB. Of the three persons who died with TB during the COVID-19 period, one had a negative virologic test, and two were not tested.

Our study outcome indicators presented important statistical differences. First, during the study period six new TB diagnoses were made (9.2\%), significantly fewer than the 15 new diagnoses $(19.7 \%)$ in the control period ( $p=0.04$ ). Second, the proportion of patients lost to follow-up was significantly higher in the study period compared to the control $(10.8 \%$ versus $2.6 \% ; \mathrm{p}=0.03)$. Third, three deaths occurred during the study (4.6\%), compared to none in the control period $(\mathrm{p}=0.04)$.

In detail, in the 2020 cohort seven of 65 patients (10.8\%) patients were lost to follow-up, three (4.6\%) had died, 10 had completed treatment (15.4\%) and 45 (69.2\%) were still engaged in care. In the control cohort, out of 76 patients, two patients were lost to follow-up (2.6\%), none had died, 11 had completed treatment $(14.5 \%)$ and $62(81.5 \%)$ were still on TB treatment (figure 1$)$.

\section{@ERSpublications}

It is important to recognise the impact of COVID-19 on TB care to mitigate its consequences. TB services have an urgent need to identify novel strategies to ensure the continuum of TB care at the time of the coronavirus pandemic. https://bit.ly/2OIKnwL

Cite this article as: Magro P, Formenti B, Marchese V, et al. Impact of the SARS-CoV-2 epidemic on tuberculosis treatment outcome in Northern Italy. Eur Respir J 2020; 56: 2002665 [https://doi.org/10.1183/ 13993003.02665-2020]. 
FIGURE 1 Tuberculosis (TB) treatment outcome during the coronavirus disease 2019 (March to April 2020) and control (March to April 2019) periods.

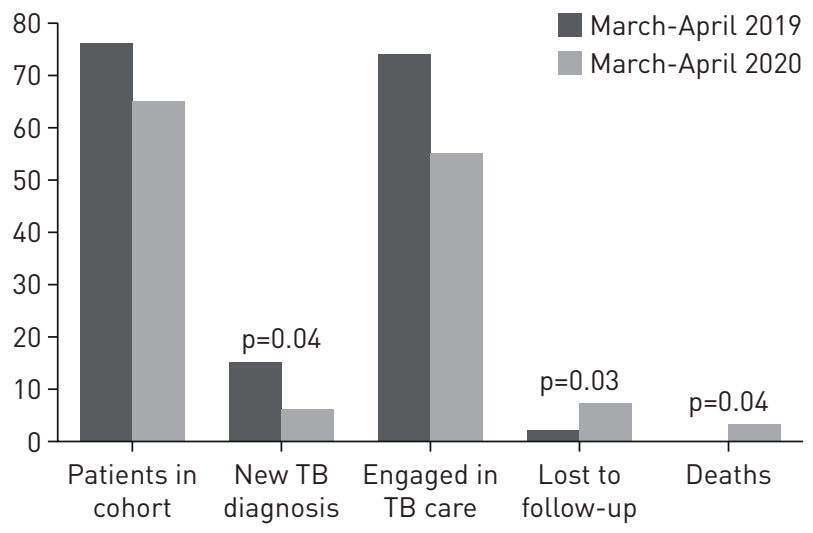

These data show that during the peak of COVID-19 epidemic our TB centre experienced a significant reduction of the number of $\mathrm{TB}$ diagnoses and an increase in the proportion of patients who were lost to follow-up or died, despite our efforts to keep TB services fully functional.

Although the potential negative impact of COVID-19 on TB services is fully recognised, data on TB treatment outcome are still limited. Some studies reported an increase in TB fatality rate associated with COVID-19 co-infection [1-3]. One study performed in Sierra Leone [5] showed trends in TB diagnosis similar to the one observed in Brescia. Besides, viral infections may have a detrimental impact on access to $\mathrm{TB}$ diagnosis and care, as recently summarised by $\mathrm{ONG}_{\mathrm{NG}}$ et al. [6]. Reasons for the reduction in TB diagnosis may include decreased attention to TB by healthcare systems, difficulties in accessing health services, lockdown measures, and fear of stigma and contagion. The same reasons may justify the increase in the proportion of cases who are lost to follow-up.

Our report has limitations, mainly the small sample size and the short observation period. However, this is the first report showing significant worsening in TB treatment outcome during the COVID-19 epidemic, supporting the hypothesis that the pandemic may seriously jeopardise TB diagnosis, care and management [7-9].

TB services have an urgent need to identify novel strategies to ensure the continuum of TB care at the time of the COVID-19 pandemic. The implementation of video observation therapy [10] may be a useful tool to ensure engagement to treatment during emergency periods. Furthermore, awareness campaigns targeting the whole health sector on the possible overlap of the clinical picture between COVID-19 and TB should be implemented to avoid diagnostic delays.

To conclude, it is important to recognise the impact of COVID-19 on TB care to mitigate its consequences. Essential health services should be maintained at their higher capacity level, even during exceptional events. Developing and strengthening policies to rapidly adapt TB control measures to the new challenge is a high and immediate priority.

Paola Magro ${ }^{1,2,6}$, Beatrice Formenti ${ }^{3,6}$, Valentina Marchese $^{2,4}$, Maurizio Gulletta ${ }^{5}$, Lina Rachele Tomasoni ${ }^{5}$, Silvio Caligaris ${ }^{5}$, Francesco Castelli ${ }^{1,2}$ and Alberto Matteelli ${ }^{1,2}$

${ }^{1}$ Dept of Infectious and Tropical Diseases, University of Brescia, ASST Spedali Civili, Brescia, Italy. ${ }^{2}$ WHO Collaborating Centre for TB/HIV and TB elimination, Brescia, Italy. ${ }^{3}$ Dept of Infectious Diseases, ASST Spedali Civili of Brescia, Brescia, Italy. ${ }^{4}$ Specialist Consultation Service, ASST Spedali Civili of Brescia, Brescia, Italy. ${ }^{5}$ Unit of Tropical Diseases, ASST Spedali Civili, Brescia, Brescia, Italy. ${ }^{6}$ These authors contributed equally.

Correspondence: Beatrice Formenti, Dept of Infectious Diseases, ASST Spedali Civili of Brescia, 25125 Brescia, Italy. Email: beatriceformenti1992@gmail.com

Received: 7 July 2020 | Accepted after revision: 15 July 2020

Conflict of interest: P. Magro has nothing to disclose. B. Formenti has nothing to disclose. V. Marchese has nothing to disclose. M. Gulletta has nothing to disclose. L.R. Tomasoni has nothing to disclose. S. Caligaris has nothing to disclose. F Castelli reports acting as a principle investigator of company-sponsored clinical trials in the field of HIV infection (ViiV Healthcare, GlaxoSmithKline, Gilead Sciences and Janssen Cilag). A. Matteelli has nothing to disclose.

Support statement: This research was carried out as part of the European Health Program 2014-2020, project: "E-DETECT, early detection and integrated management of tuberculosis in Europe". Funding was received from the European Commission (709624). 


\section{References}

1 Tadolini M, Codecasa LR, García-García JM, et al. Active tuberculosis, sequelae and COVID-19 co-infection: first cohort of 49 cases. Eur Respir J 2020; 56: 2001398.

2 Stochino C, Villa S, Zucchi P, et al. Clinical characteristics of COVID-19 and active tuberculosis co-infection in an Italian reference hospital. Eur Respir J 2020; 56: 2001708.

3 Motta I, Centis R, D’Ambrosio L, et al. Tuberculosis, COVID-19 and migrants: preliminary analysis of deaths occurring in 69 patients from two cohorts. Pulmonology 2020; 26: 233-240.

4 Dipartimento della Protezione Civile, Data on COVID-19. http://opendatadpc.maps.arcgis.com/apps/ opsdashboard/index.html\#/b0c68bce2cce478eaac82fe38d4138b1. Date last accessed: 25 June, 2020.

5 Buonsenso D, Iodice F, Sorba Biala J, et al. COVID-19 effects on tuberculosis care in Sierra Leone. Pulmonology 2020; in press [https://doi.org/10.1016/j.pulmoe.2020.05.013]

6 Ong CWM, Migliori GB, Raviglione M, et al. Epidemic and pandemic viral infections: impact on tuberculosis and the lung. A consensus by the World Association for Infectious Diseases and Immunological Disorders (WAidid), Global Tuberculosis Network (GTN) and members of the European Society of Clinical Microbiology and Infectious Diseases Study Group for Mycobacterial Infections (ESGMYC). Eur Respir J 2020; 56: 2001727.

7 Adepoju P. Tuberculosis and HIV responses threatened by COVID-19. Lancet HIV 2020; 7: e319-e320.

8 Togun T, Kampmann B, Stoker NG, et al. Anticipating the impact of the COVID-19 pandemic on TB patients and TB control programmes. Ann Clin Microbiol Antimicrob 2020; 19: 21.

9 Alagna R, Besozzi G, Codecasa LR, et al. Celebrating World Tuberculosis Day at the time of COVID-19. Eur Respir J 2020; 55: 2000650.

10 Story A, Aldridge RW, Smith CM, et al. Smartphone-enabled video-observed versus directly observed treatment for tuberculosis: a multicentre, analyst-blinded, randomised, controlled superiority trial. Lancet; 393: 1216-1224. 